\title{
Robust and sensitive LC/MS-MS method for simultaneous detection of acetylsalicylic acid and salicylic acid in human plasma
}

Dávid Sirok ${ }^{\mathrm{a}, \mathrm{b}}$, Márta Pátfalusi ${ }^{\mathrm{a}}$, Gábor Szeleczkya ${ }^{\mathrm{a}}$, Gyula Somorjai ${ }^{\mathrm{a}}$, Dávid Greskovits ${ }^{\mathrm{c}}$, Katalin Monostory ${ }^{\mathrm{d}}$

${ }^{a}$ ATRC Aurigon Toxicological Research Center Ltd., Budapest, Hungary

${ }^{\mathrm{b}}$ Toxi-Coop Toxicological Research Center Co., Budapest, Hungary

${ }^{\mathrm{c}}$ Meditop Pharmaceutical Ltd., Pilisborosjenő, Hungary

${ }^{\mathrm{d}}$ Research Centre for Natural Sciences, Hungarian Academy of Sciences, Budapest, Hungary

Highlights:

- A LC-MS/MS method was developed and validated for simultaneous determination of aspirin and salicylic acid in human plasma.

- The accuracy and the precision of the method with one-step liquid-liquid extraction were excellent for both analytes.

- The method was highly robust and suitable for the analysis of $>2000$ samples in a pharmacokinetic study.

- The present method can contribute to the improvement of ASP/SAL determination in patients under antithrombotic therapy.

- The method can contribute to the reduction of the risk for ASP resistance associated with bioavailability/exposure issues. 


\begin{abstract}
A liquid chromatography-tandem mass spectrometry method was developed for the simultaneous determination of acetylsalicylic acid (ASP) and its major metabolite, salicylic acid (SAL) in human plasma. The method was validated. ASP and SAL were extracted by single-step liquid-liquid extraction using tert-butyl methyl ether. The ions were detected in multiple reaction monitoring mode at $\mathrm{m} / \mathrm{z}$ 179.0/137.0 transition for ASP and $\mathrm{m} / \mathrm{z}$ 136.9/93.0 for SAL. The lower limit of quantification for ASP and SAL was 1 and $80 \mathrm{ng} / \mathrm{ml}$, respectively. The method was successfully applied for the characterization of the plasma concentration levels of ASP and SAL after oral administration of Aspirin Protect 100 to healthy volunteers. The present method can contribute to the improvement of ASP and SAL determination in patients under antithrombotic therapy, and to the reduction of the risk for ASP resistance associated with bioavailability/exposure issues (non-compliance, misdosing, poor absorption).
\end{abstract}

\title{
Keywords
}

aspirin, salicylic acid, LC/MS-MS, validation, human plasma 


\section{Introduction}

Aspirin (ASP) is a well-known salicylate drug (Fig. 1), which is one of the least expensive and most widely used anti-inflammatory, analgesic and antipyretic agent [1]. In addition, ASP at low doses ( $<100 \mathrm{mg} /$ day) is applied for thrombosis prophylaxis, because it selectively inhibits cyclooxygenase (COX)-dependent platelet aggregation [2]. The mechanism of action and the effects of ASP depend on the dose taken [3]. At low doses (30$100 \mathrm{mg} /$ day), ASP irreversibly acetylates COX-1, which is responsible for the synthesis of prostanoids and for the generation of thromboxane A2. The antithrombotic effect of ASP is attributed to the selective inhibition of COX-1 dependent production of thromboxane A2 in platelets [4,5]. High-dose ASP (2-4 g/day) inhibits both COX-1 and COX-2, blocks prostaglandin production, and displays anti-inflammatory, antipyretic and analgesic effects. At extremely high doses (6-8 g/day), ASP has been reported to be as effective as cortisone in treatment of rheumatic disorders [6]. ASP has a short half-life, approximately 15-20 min in plasma, because it is rapidly hydrolyzed by esterases to salicylic acid (SAL) (Fig. 1) [7,8]. SAL is metabolized to gentisic acid or biotransformed by conjugation with glycin or glucuronic acid [7]. SAL also displays some anti-inflammatory, antipyretic and analgesic activity; however, the pharmacological activity of SAL is far from that of the parent compound [9]. The efficacy and safety aspects of ASP therapy require the establishment of the optimal dose for the particular indication. Therefore, optimization of ASP dosing is essential for the avoidance of both adverse drug reactions and ASP resistance. Misdosing of patients appears to be rather frequent; $10-20 \%$ of patients with cardiovascular disease are resistant to ASP [10]. ASP resistance is the inability of ASP to protect patients from thrombotic complications, to cause a prolongation of the bleeding time, or to inhibit thromboxane biosynthesis [11,12]. One of the possible reasons of ASP failure is the reduced bioavailability of ASP which is induced by inadequate intake of ASP, inadequate ASP dosing, and reduced absorption or increased metabolism of ASP [12].

The bioavailability of ASP can be monitored by the simultaneous determination of ASP and SAL plasma levels in patients, and the optimal ASP dosing can be tailored individually. Hereby the risk of the clinical atherothrombotic vascular events in patients can be reduced. In addition, the guideline of the European Medicines Agency on the investigation of bioequivalence requires the determination of plasma levels of both ASP and SAL for a new ASP formulation since 2010 [13]. For the measurement of salicylates, several techniques are 
available, such as colorimetric, fluorometric, enzymatic, gas or liquid chromatographic assays [14]. Bioanalytical HPLC-UV methods have also been reported for the quantification of ASP and its metabolites [15-21]. However, the liquid chromatography-tandem mass spectrometry (LC-MS/MS) offers the most sensitive and selective methods for measuring salicylates simultaneously from biological matrices. The number of the published LC-MS/MS methods that are suitable for the simultaneous determination of ASP and SAL from plasma is still limited. Bae et al. [22] applied sample preparation methods separately for the parent compound and its deacetylated metabolite, whereas others reported multi-step sample preparation (protein precipitation followed by a liquid-liquid extraction) [23], one-step solidphase extraction [24], or even direct injection of the supernatant of precipitated plasma into the LC-MS/MS system [25]. To the best of our knowledge, no published LC-MS/MS method with a one-step liquid-liquid extraction is available for the simultaneous extraction and quantification of ASP and SAL in human plasma. The main advantages of the present method over the previously published methods are the single-step liquid-liquid extraction, the better retention on the HPLC column to lower the risk of matrix effect, the more symmetric peak shapes and the inexpensive materials necessary. The aim of the current work has been the development of a sensitive, selective and economical LC/MS-MS method for assaying ASP resistance in patients.

\section{Experimental}

\subsection{Chemicals and reagents}

ASP and SAL (analytical standards) were purchased from Sigma-Aldrich Inc. (St Louis, MO, USA), while ASP-d4 and SAL-d4 (2-hydroxybenzoic-3,4,5,6-d4 acid) (internal standards) were obtained from Toronto Research Chemicals Inc. (Toronto, Canada) and C/D/N Isotopes Inc. (Pointe-Claire, Canada), respectively. Tert-butyl methyl ether (TBME) (LiChrosolv) and acetic acid (glacial, 100\% Suprapur) were procured from Merck Inc. (Darmstadt, Germany). Formic acid (puriss p.a., 98-100\%) and potassium fluoride (ACS reagent, $\geq 99.0 \%$ ) were purchased from Sigma-Aldrich Inc. Acetonitrile (ultra gradient HPLC grade) was from J.T. Baker (Deventer, The Netherlands). Blank human plasma was purchased from Sera Laboratories International Ltd. (Haywards Heath, United Kingdom). 


\subsection{Chromatographic conditions}

The Agilent 1100 series HPLC system consisted of a G1312A binary pump, a G1322A degasser, a G1329A autosampler and a G1330A autosampler thermostat. The HPLC column was an InertSustain C18 $(2.1 \times 75 \mathrm{~mm}, 3 \mu \mathrm{m})$ (GL Sciences Inc., Nishi Shinjuku, Japan), kept at ambient temperature $\left(23 \pm 3^{\circ} \mathrm{C}\right)$. Other HPLC columns tested for separation

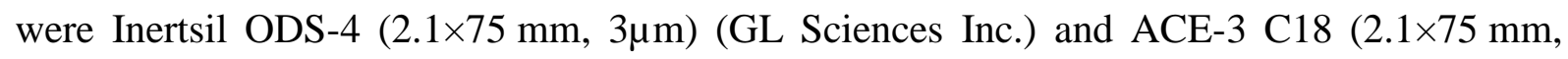
3 $\mu \mathrm{m}$ ) (Advanced Chromatography Technologies Ltd., Aberdeen, UK). The HPLC mobile phases were Milli-Q water containing $0.2 \%$ formic acid (eluent A) and acetonitrile containing $0.2 \%$ formic acid (eluent B). The analytes were eluted by a gradient program. After sample injection ( 0 min), a mixture of $80 \%$ eluent $\mathrm{A}$ and $20 \%$ of eluent $\mathrm{B}$ was linearly changed to a mixture of $35 \%$ eluent A and $65 \%$ of eluent B until 4.0 min. Then eluent B was linearly reversed back to $20 \%$ at $4.1 \mathrm{~min}$. Finally, the initial combination of the eluents was kept up to $6.5 \mathrm{~min}$ for equilibration of the column. The flow rate was set at $0.3 \mathrm{ml} / \mathrm{min}$, and the injected sample volume was $10 \mu \mathrm{l}$. A diverter valve was used to discard the LC effluent during the first $2.5 \mathrm{~min}$ and the last $1.0 \mathrm{~min}$ of each chromatographic run.

\subsection{Mass spectrometric conditions}

The mass spectrometer was an API2000 triple quadrupole instrument equipped with an electrospray ionization (ESI) interface (Applied Biosystems, Toronto, Canada). Data were acquired and processed by the Analyst software (version 1.4.2). The source parameters, curtain gas, collision gas, ion spray voltage, source temperature, nebulizer gas and auxiliary gas were set at $30 \mathrm{psi}, 3 \mathrm{psi},-500 \mathrm{~V}, 400{ }^{\circ} \mathrm{C}, 60 \mathrm{psi}$ and $60 \mathrm{psi}$, respectively. The parameters of the mass spectrometer, declustering potential, entrance potential, collision energy, collision entrance potential and collision exit potential for ASP and ASP-d4 were $-15 \mathrm{~V},-10 \mathrm{~V},-10 \mathrm{~V},-10 \mathrm{~V}$ and $-4 \mathrm{~V}$, respectively. These parameters for SAL and SAL-d4 were $-60 \mathrm{~V},-15 \mathrm{~V},-42 \mathrm{~V},-15 \mathrm{~V}$ and $-4 \mathrm{~V}$, respectively. Detection of the ions was performed in multiple reaction monitoring (MRM) mode, monitoring the transition of the $\mathrm{m} / \mathrm{z} 179.0$ parent ion to the $\mathrm{m} / \mathrm{z} 137.0$ product ion for ASP, the $\mathrm{m} / \mathrm{z} 136.9$ parent ion to the $\mathrm{m} / \mathrm{z} 93.0$ product ion for SAL, the $\mathrm{m} / \mathrm{z} 183.0$ parent ion to the $\mathrm{m} / \mathrm{z} 141.0$ product ion for ASP-d4 and the $m / z 140.9$ parent ion to the $m / z$ 97.0 product ion for SAL-d4. The dwell time was $400 \mathrm{~ms}$ for all components. 


\subsection{Sample preparation}

The primary stock solutions of ASP and SAL for the preparation of calibrator (STC) and quality control (STQ) samples were prepared by separate weighing (MX5 balance, Mettler-Toledo International Inc., Switzerland). The primary stock solutions of ASP, SAL

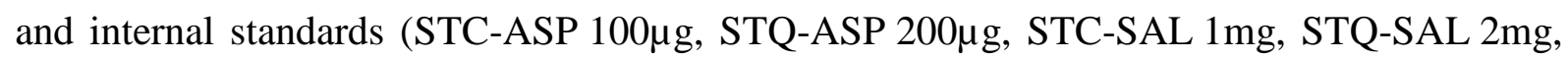

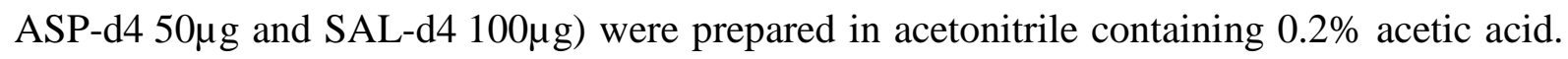
The working solutions used for spiking the calibrator and quality control (QC) samples were diluted separately by spiking an appropriate volume of the stock solutions to achieve the final concentrations for ASP, SAL and the internal standards. The dilutions were performed in acetonitrile containing $0.2 \%$ acetic acid. Stock and working solutions were stored at $-20 \pm 5{ }^{\circ} \mathrm{C}$.

A simple liquid-liquid extraction method was followed for the extraction of ASP and SAL from human plasma. Because of the instability of ASP in plasma, potassium oxalate and sodium fluoride were used as anticoagulant, and the sample preparation was performed in icewater bath. The enzymes in human plasma were inhibited by $12 \%$ formic acid and $450 \mathrm{mg} / \mathrm{ml}$ potassium fluoride solution. The plasma and the $12 \%$ formic acid solution were mixed in 10:1 volume ratio. An aliquot of $275 \mu \mathrm{l}$ acidified human plasma, $10 \mu \mathrm{l}$ of $450 \mathrm{mg} / \mathrm{ml}$ potassium fluoride solution, $10 \mu \mathrm{l}$ of the corresponding standard working solution and $10 \mu \mathrm{l}$ of IST solution were mixed. $2 \mathrm{ml}$ of TBME was added to the samples, mixed (Vortex mixer, Velp Scientifica, England) and shaken (Multi-Tube vortexer, VWR International, USA) for $10 \mathrm{~min}$. The samples were centrifuged (Harrier 18/80R centrifuge, Sanyo Electric Co., Japan) at $3,500 \times \mathrm{g}$ for $5 \mathrm{~min}$ at $4{ }^{\circ} \mathrm{C}$. The upper phase $(1.6 \mathrm{ml})$ was evaporated (Turbo Vap LV evaporator, Zymark Co., USA) and the evaporation residue was reconstituted in $250 \mu \mathrm{l}$ of Milli-Q water (ELIX 3/Gradient A10 water purification system, Millipore Ltd., France) containing $0.2 \%$ acetic acid.

\subsection{Validation of the analytical method}

Validation of the procedure was performed in order to evaluate the method in terms of accuracy, precision, selectivity, sensitivity, linearity, recovery, matrix effect including the influence of haemolysis, carry-over, dilution effect and stability. The acceptance criteria were determined according to the FDA and EMEA guidance for bioanalytical method validation [26,27]. 


\subsubsection{Selectivity}

Solutions of ASP and SAL were injected with the peak areas thereof equal to the nonextracted highest calibrator. Solutions of ASP-d4 and SAL-d4 internal standards (IST) were injected with the peak areas thereof equal to that of IST in the samples. Each selectivity solution was injected separately to ensure that the analytes or ISTs did not contain any interfering impurity erroneously detected as the other compound. As a reference, for the quantification of any interference a lower limit of quantification (LLOQ) sample was also injected. Six blank human plasma samples from different lots (obtained under controlled conditions from different individuals) were tested for interference. The results were compared to those ones obtained for the LLOQ plasma sample.

\subsubsection{Calibration curve}

The nine-point calibration curves (for ASP: 1, 2, 5, 10, 20, 50, 100, 200 and $500 \mathrm{ng} / \mathrm{ml}$; and for SAL: 80, 160, 240, 320, 800, 1600, 2400, 3200, $8000 \mathrm{ng} / \mathrm{ml}$ ) were constructed on the basis of the response ratio of the analyte to the corresponding IST versus the ratio of the analyte concentration to the corresponding IST concentration. The concentrations of ISTs in spiked plasma samples were $40 \mathrm{ng} / \mathrm{ml}$ for ASP-d4 and $400 \mathrm{ng} / \mathrm{ml}$ for SAL-d4. The weighting type was set to $1 / \mathrm{y}^{2}$.

\subsubsection{Accuracy and precision}

The intra-day accuracy and precision were determined by analyzing six replicates of ASP and SAL at four different QC levels in human plasma. The inter-day precision was determined by analyzing the four levels of QC samples in 3 different runs.

\subsubsection{Matrix effect and recovery}

For the investigation of matrix effect, the following samples were analyzed: Six different human plasma lots were used to prepare QC high and QC low samples ( $\mathrm{n}=6$ for each concentration) with matrix. Post-extraction spiked samples were obtained by spiking blank plasma extract with the respective working solution. Matrix-free reference solutions were prepared at the concentration of the high and low QC samples, six each.

For each individual plasma, the IST normalized matrix factor was calculated as follows (1, 2 and 3): 


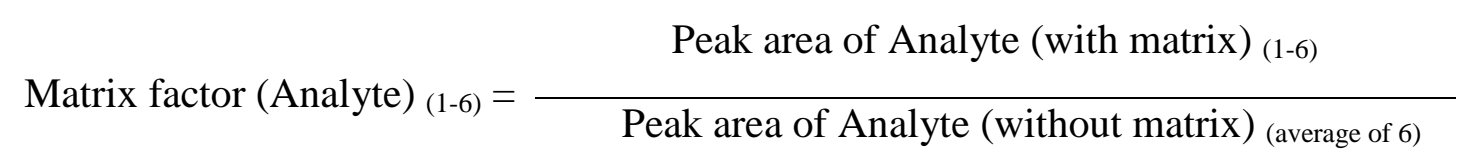

$$
\text { Matrix factor }(\text { IST) })_{(1-6)}=\frac{\text { Peak area of IST (with matrix) })_{(1-6)}}{\text { Peak area of IST (without matrix) } \text { (average of 6) }_{\text {(2) }}}
$$

$$
\text { Matrix factor (IST normalized) })_{(1-6)}=\frac{\text { Matrix factor of Analyte }_{(1-6)}}{\text { Matrix factor of IST }_{(1-6)}}
$$

For the evaluation of matrix effect, the coefficient of variation (CV\%) of the IST normalized matrix factors was calculated. The matrix effect on ionisation was accepted if the coefficient of variation (CV\%) of IST normalized matrix factors was within 15\%.

For the determination of extraction recovery, the six different plasma lots (used for the determination of matrix effect) were investigated. The peak area of the analyte obtained for extracted blank samples spiked with the low and high QC solutions was compared to the peak area of the analyte obtained for the corresponding processed QC samples.

$$
\text { Recovery [\%] }=\quad \frac{(\text { Peak area of the extracted QC sample }) \times 100}{\text { Peak area of the spiked blank extract }}
$$

For calculation of the recovery (4), the signal of the individual QC samples was compared to the mean of the reference samples. Finally, the individual recovery values were averaged. Acceptance criteria: The recovery optimally is $>80 \%$. Lower than $80 \%$ recovery could also be accepted on condition the precision for the six different blanks did not exceed 15\%. For the internal standards, the mean peak areas obtained for the QC samples were compared to those of the neat solutions reflecting the combined effect of the matrix and recovery.

\subsubsection{Influence of haemolysis}

For investigation of the potential effect of the haemolysis, QC samples were prepared in haemolysed plasma. Blank human oxalate/fluoride blood was collected freshly. The sample 
was intensively mixed until haemolysis. $1.95 \mathrm{ml}$ of blank oxalate/fluoride plasma was mixed with $0.05 \mathrm{ml}$ of haemolysed human blood and then the sample was acidified. From this matrix, 6 replicates of QC samples at the medium concentration were prepared and analyzed.

\subsubsection{Stability}

Stability tests were conducted to evaluate the stability of the analytes in standard solutions, in whole blood and in plasma samples under different conditions. The stability of the analytes in the spiking solutions used for the preparation of the low and high QC samples was investigated after storage for 4 hours at room temperature and after 33-day and 90-day storage at $-20 \pm 5{ }^{\circ} \mathrm{C}$ in a deep freezer. The stability in whole blood stored in ice-water bath (1 hour) was performed by comparing the measured concentrations of the analytes in the stability samples with those in the freshly prepared samples. Short-term plasma stability in ice-water bath (4 hours), autoinjector stability (52 hours), freeze-thaw stability (3 cycles), long-term plasma stability (33 and 90 days at $-70{ }^{\circ} \mathrm{C}$ ) and re-injection stability (124 hours) tests were performed at the low and high QC levels.

\subsubsection{Dilution test}

To extend the upper limit of calibration, the possibility of dilution was investigated for samples containing ASP and SAL at high concentrations. Diluted high QC samples were prepared at the concentration of $2,000 \mathrm{ng} / \mathrm{ml}$ for ASP and 32,000 $\mathrm{ng} / \mathrm{ml}$ for SAL. After the applied five times dilution, the concentrations of the diluted QC samples corresponded to the concentrations of the high QC samples.

\subsection{Pharmacokinetic analysis}

In the pharmacokinetic study, 33 healthy, mixed gender volunteers (18 to 45 years of age) were enrolled. The study protocol was approved by the National Institute of Pharmacy and Nutrition (Budapest, Hungary). For investigations, informed consent was obtained from the participants. The volunteers were administered orally by a single-dose Aspirin Protect $100 \mathrm{mg}$ enteric coated tablet (Bayer Pharma AG) containing $100 \mathrm{mg}$ of ASP. Volunteers were fasted for at least 10 hours before and 4 hours after drug administration. They were given standard diet 4, 6 and 12 hours after drug administration. The tablet was taken with $200 \mathrm{ml}$ of tap water. For all subjects, the following blood samples were taken: predose (within $60 \mathrm{~min}$ ) and at post-dose 0.50, 1.00, 1.50, 2.00, 2.50, 3.00, 3.50, 4.00, 4.50, 5.00, $5.50,6.00,6.50,7.00,7.50,8.00,8.50,9.00,10.00,11.00,12.00,13.00,14.00,16.00$ and 
24.00 hours. Blood samples (approximately $5 \mathrm{ml}$ ) were taken via an indwelling catheter from the forearm vein into a vacutainer tube. The blood samples were collected in tubes containing potassium oxalate and sodium fluoride as anticoagulant and esterase inhibitor, gently homogenized and cooled immediately in an ice-water bath until centrifugation. The blood samples were centrifuged within $30 \mathrm{~min}$ after blood taking at $1500 \times \mathrm{g}$ at $4{ }^{\circ} \mathrm{C}$ for $10 \mathrm{~min}$ to obtain plasma. Immediately after centrifugation, $1.5 \mathrm{ml}$ of plasma was transferred into a polypropylene tube containing $0.15 \mathrm{ml}$ of $12 \%$ formic acid solution in water to protect ASP from chemical hydrolysis. The tubes were capped, frozen immediately in dry ice and stored frozen (below $-70^{\circ} \mathrm{C}$ ) until the analysis. 


\section{Results and discussion}

\subsection{Optimizing the analytical system}

Optimization of the chromatographic method for the simultaneous determination of ASP and SAL in human plasma was challenging. i) ASP and SAL are polar compounds; therefore, their retention in conventional RP columns (C18, C8, phenyl stationary phases) is rather poor. ii) Complete separation of the analytes is essential, because ASP undergoes source fragmentation in the mass spectrometer and forms SAL. iii) In addition, highly different calibration ranges are required for ASP and SAL because of the endogenous SAL levels in blank human plasma $[28,29]$. iv) In the case of partial separation of the analytes, the much higher SAL levels can lead to ion suppression in the source of the mass spectrometer.

The chromatographic separation was tested on several highly hydrophobic and inert HPLC columns (InertSustain C18, Inertsil ODS-4, ACE-3 C18) using various combinations of acetonitrile, methanol and additives (acetic acid, formic acid, ammonium formate buffer). The best retention, peak shapes and chromatographic resolution were achieved on the InertSustain C18 column with a flow rate of $0.3 \mathrm{ml} / \mathrm{min}$ and a total run time of $6.5 \mathrm{~min}$. The best suitable mobile phase system for the gradient method was Milli-Q water and acetonitrile containing $0.2 \%$ formic acid. For optimization of the mass spectrometric parameters, a full scan mass spectrum was recorded for both ASP and SAL in negative ionization mode with electrospray ionization source. Multiple reaction monitoring scanning mode was used to obtain the best selectivity. No significant solvent adduct ions were observed. To detect as low as $1 \mathrm{ng} / \mathrm{ml}$ ASP level in human plasma, the maximal sensitivity of the mass spectrometer was required, whereas the limit of quantification for SAL was 80 times higher than for ASP. To prevent the ion saturation of the detector, non-optimal tuning parameters were used for the determination of SAL. Although the optimal declustering potential and collision energy for SAL would have been $-28 \mathrm{~V}$ and $-20 \mathrm{~V}$, we used the parameters of $-60 \mathrm{~V}$ and $-42 \mathrm{~V}$, respectively.

\subsection{Validation of the analytical method}

\subsubsection{Selectivity and specificity}

The present method was selective, since no endogenous interfering peak was observed for ASP in the individual and pooled blank human plasma samples. However, trace levels of 
SAL originating from the nutrients could not be avoided in the blank samples $[28,29]$. The retention times were stable, approximately $3.65 \mathrm{~min}$ for ASP and ASP-d4, and $4.0 \mathrm{~min}$ for SAL and SAL-d4, respectively. No carry-over was observed. The typical MRM chromatograms for ASP and SAL are shown in Fig. 2.

\subsubsection{Linearity}

The 9-point calibration curves proved to be linear in the $1-500 \mathrm{ng} / \mathrm{ml}$ concentration range for ASP and in the $80-8,000 \mathrm{ng} / \mathrm{ml}$ concentration range for SAL. The LLOQ was $1 \mathrm{ng} / \mathrm{ml}$ for ASP and $80 \mathrm{ng} / \mathrm{ml}$ for SAL. The mean correlation coefficient (r) of the respective weighted calibration curves generated during the validation were 0.9985 (ranged between 0.9974 and 0.9995 ) for ASP, and 0.9992 (ranged between 0.9986 and 0.9997 ) for SAL.

\subsubsection{Accuracy and precision}

The intra- and inter-day accuracy and precision data for ASP and SAL are presented in Table 1 . The method fulfilled the postulated acceptance criteria concerning the intra-day and inter-day accuracy and precision defined in the FDA and EMEA guidances [26,27].

\subsubsection{Extraction recovery, matrix effect, influence of haemolysis and dilution} effect

The extraction recovery was determined by comparing extracted QC samples and post-spiked QC samples. The mean recoveries in the six individual blank human plasma for ASP were $88.4 \pm 6.5 \%$ at the concentration of $3 \mathrm{ng} / \mathrm{ml}$ and $91.1 \pm 3.7 \%$ at the concentration of $400 \mathrm{ng} / \mathrm{ml}$, whereas for SAL were $70.1 \pm 6.2 \%$ at the concentration of $240 \mathrm{ng} / \mathrm{ml}$ and $82.3 \pm 4.2 \%$ at the concentration of $6,400 \mathrm{ng} / \mathrm{ml}$. A negligible matrix effect was observed for both analytes and the internal standards. The CV\% values of IST normalized matrix factors were $6.71 \%$ and $3.81 \%$ at the concentration of low QC and high QC samples for ASP, and 7.09\% and 3.07\% for SAL, respectively. The combined effect of the matrix and the recovery for ASP-d4 and SAL-d4 was 90.5\% and 84.1\%, respectively.

The peak area ratios obtained for the high and low QC samples were compared for pure plasma and plasma sample containing $2.5 \%$ haemolyzed blood. The haemolysis had no influence on the reliability of the quantification. The deviation for the standard/IST peak area ratios remained below the $\pm 15 \%$ acceptance limit (deviation: $+9.73 \%$ for ASP and $-3.45 \%$ for SAL). 
The upper concentration limits can be extended to 2,500 ng/ml for ASP and 40,000 $\mathrm{ng} / \mathrm{ml}$ for SAL by 5 times dilution with blank human plasma. The mean deviation from the nominal concentration was $-11.5 \%$ and $-9.4 \%$, while the precision $5.0 \%$ and $3.7 \%$ for ASP and SAL, respectively.

\subsubsection{Stability}

In the different stability experiments carried out, viz. short-term plasma in ice-water bath, repeated freeze-thaw (3 cycles), autosampler, long-term plasma, whole blood, short-term benchtop solution and long-term solution, the results were found to be within the acceptable limits during the entire validation. The analytes and ISTs proved to be stable in working solutions for at least 4 hours at room temperature and 90 days in a freezer $\left(-20 \pm 5{ }^{\circ} \mathrm{C}\right)$. Both ASP and SAL proved also to be stable in oxalate/fluoride human blood for at least 1 hour and acidified oxalate/fluoride human plasma for at least 4 hours kept in an ice-cooled water bath. ASP and SAL were also stable in frozen plasma during 3 freeze/thaw cycles and storage in an ultra-freezer for at least 90 days (below $-70^{\circ} \mathrm{C}$ ). The plasma extract can be stored in the autoinjector at $4{ }^{\circ} \mathrm{C}$ for at least 52 hours prior to analysis. The samples can be re-injected together with the calibrators after storage at $+4{ }^{\circ} \mathrm{C}$ for up to 124 hours. The main results of stability tests are shown in Table 2.

\subsection{Evaluation of the present analytical method}

Comparing to the published methods that are available for the simultaneous determination of ASP and SAL from human plasma, the present method was as sensitive as the most sensitive one in the literature [24]. In the method published by Xu et al. [25], protein precipitation by acidified acetonitrile was applied as sample preparation. It was a very simple procedure, however, the extraction recovery was variable over the concentration range. In the method described by Bae et al. [22], the samples for ASP and SAL were prepared separately by liquid-liquid extraction and protein precipitation, respectively. As this method used duplicate sample preparation for the analysis of the analytes, it was time consuming for both the sample preparation and the analysis. Nirogi et al. [23] developed a two-step extraction procedure for the simultaneous extraction of ASP and SAL. They used a protein precipitation method followed by a solvent extraction step for sample preparation. It produced consistent recovery over the measured concentration range; however, it was a two-step method and the 
average recoveries were only $45 \pm 2.4 \%$ and $40 \pm 1.8 \%$ for ASP and SAL, respectively. Bharathi et al. [24] extracted ASP and SAL simultaneously from human plasma by a solid-phase extraction method. This method resulted in high extraction recovery with low variability over the detected concentration range (76.5-99.4\% for ASP, and 72.6-101.5\% for SAL); however, it was a less cost-effective sample preparation method due to the material necessary (SPE cartridges, organic solvents for the equilibration, washing and elution). The extraction recoveries in the present method by the one-step liquid-liquid extraction were at least as high and consistent as by the best working sample preparation method (solid-phase extraction). All of the organic solvent needed was $2 \mathrm{ml}$ of TBME for one sample. Additionally, the method was highly robust, and suitable for the analysis of more than 2000 samples in a pharmacokinetic study. Thanks to the clean sample extracts and to the column washing step in the chromatographic gradient method, the matrix effect was negligible during the analysis of the samples throughout. Comparing the length of the stability of ASP in human plasma published in the literature [30], the present method provided the longest stability period (ASP was stable below $-70^{\circ} \mathrm{C}$ for at least 90 days).

\subsection{Pharmacokinetics in patients}

The present method was applied for the analysis of plasma samples obtained from 33 healthy volunteers following oral administration of Aspirin Protect $100 \mathrm{mg}$ enteric coated tablet. The mean plasma concentration - time profiles for ASP and SAL are shown in Fig. 3, and some relevant pharmacokinetic parameters are presented in Table 3.

Based on the sensitivity and robustness, the method was found to be suitable for the accurate characterization of the plasma level curves for ASP and SAL in human. The variability of the results for ASP was approximately twice as high as for SAL. The most extreme pharmacokinetic curves are shown in Fig. 4. These volunteers presented about one order of magnitude difference between the $C_{\max }$ values of the plasma level curves for ASP (Table 4), whereas less than $20 \%$ difference for SAL. The high variability of the plasma concentration levels for ASP can account for the inter-individual differences in the bioavailability of ASP which can be the source of the variability in pharmacological effects in patients. Furthermore, the simultaneous determination of ASP and SAL plasma levels can also reveal some reasons of ASP inefficiency, because the presence of SAL can prevent or even reverse the platelet function inhibiting the effect of ASP [31]. 


\section{Conclusions}

A robust and sensitive LC-MS/MS method was developed and validated for the simultaneous determination of ASP and SAL in human plasma. Different calibration ranges were required for the analytes because of the endogenous SAL levels in blank human plasma, therefore non-optimal MS tuning parameters were used for the determination of SAL. The application of the non-optimal tuning parameters had no impact on the stability of the signal. The accuracy and the precision of the analytical method were excellent for both the parent compound and its deacetylated metabolite. All of the organic solvent required for the one-step liquid-liquid extraction was only $2 \mathrm{ml}$ of TBME for one sample. The one-step sample preparation method as well as the robustness and specificity of the assay simplified the characterization of ASP and SAL plasma levels in volunteers. The present method can contribute to the improvement of ASP/SAL determination in patients under antithrombotic therapy and to the reduction of the risk for ASP resistance associated with bioavailability/exposure issues (non-compliance, underdosing, poor absorption). 


\section{References}

[1] J.R. Vane, R.M. Botting, The mechanism of action of aspirin, Thromb. Res. 110 (2003) 255-258.

[2] J.W. Burch, N. Standford, P.W. Majerus, Inhibition of platelet prostaglandin synthetase by oral Aspirin, J. Clin. Invest. 61 (1978) 314-319.

[3] M.H. Pillinger, C. Capodici, P. Rosenthal, N. Kheterphal, S. Hanft, M.R. Philips, G. Weissmann, Modes of action of aspirin-like drugs: Salicylates inhibit Erk activation and integrin-dependent neutrophil adhesion, Proc. Natl. Acad. Sci.: Pharmacology 95 (1998) 14540-14545.

[4] J.W. Eikelboom, J. Hirsh, F.A. Spencer, T.P. Baglin, J.I. Weitz, Antiplatelet drugs: Antithrombotic Therapy and Prevention of Thrombosis, 9th ed: American College of Chest Physicians Evidence-Based Clinical Practice Guidelines, Chest 141(2 Suppl) (2012) e89S-119S.

[5] R. Casado-Arroyo, C. Sostres, A. Lanas, Optimizing the use of aspirin for cardiovascular prevention, Drugs 73 (2013) 803-814.

[6] I.E. Lundberg, C. Grundtman, E. Larsson, L. Klareskog, Corticosteroids--from an idea to clinical use, Best Pract. Res. Clin. Rheumatol. 18 (2004) 7-19.

[7] C.J. Needs, P.M. Brooks, Clinical pharmacokinetics of the salicylates, Clin. Pharmacokinet. 10 (1985) 164-177.

[8] T.C. Kwong, Analysis of acetylsalicylic acid and its metabolite by liquid chromatography, J. Liquid Chromatography 10 (1987) 305-321.

[9] D.E. Furst, Are There Differences Among Nonsteroidal Antiinflammatory Drugs?, Arthritis Rheum. 37 (1994) 1-9.

[10] A.D. Michelson, M. Cattaneo, J.W. Eikelboom, P. Gurbel, K. Kottke-Marchant, T.J. Kunicki, F.M. Pulcinelli, C. Cerletti, A.K. Rao, Aspirin resistance: position paper of the Working Group on Aspirin Resistance, J Thromb Haemost. 3 (2005) 1309-1311.

[11] P. Harrison, A.L. Freelinger, M.I. Furman, A.D. Michelson, Measuring antiplatelet drug effects in the laboratory, Thrombosis Research 120 (2007) 323-336.

[12] G.J. Hankey, J.W. Eikelboom, Aspirin resistance, Lancet 367 (2006) 606-617. 
[13] http://www.ema.europa.eu/docs/en_GB/document_library/Scientific_guideline/2010/0 1/WC500070039.pdf

[14] T.C. Kwong, Salicylate measurement: clinical usefulness and methodology, CRC Crit Rev Lab Med. 25 (1987) 137-139.

[15] D.C. Mays, D.E. Sharp, C.A. Beach, R.A. Kershaw, J.R. Bianchine, N. Gerber, Improved method for the determination of Aspirin and its metabolites in biological fluids by high-performance liquid chromatography: applications to human and animal studies, J. Chromatogr. B: Biomed. Sci. Appl. 311 (1984) 301-309.

[16] G.W. Peng, M.A. Gadalla, V. Smith, A. Peng, W.L. Ghiou, Simple and rapid high pressure liquid chromatographic simultaneous determination of aspirin, salicylic acid, and salicyluric acid in plasma, J. Pharm. Sci. 67 (1978) 710-720.

[17] T.C. Kwong, Analysis of acetylsalicylic acid and its metabolite by liquid chromatography, Journal of Liquid Chromatography 10 (1987) 305-321.

[18] S.K. Bakar, S. Niazi, High performance liquid chromatographic determination of aspirin and its metabolites in plasma and urine, J. Pharm. Sci. 72 (1983) 1020-1023.

[19] R.J. O’Kruk, M.A. Adams, R.B. Philip, Rapid and sensitive determination of acetylsalicylic acid and its metabolites using reversed-phase high-performance liquid chromatography, J. Chromatogr. B: Biomed. Sci. Appl. 310 (1984) 343-352.

[20] J. Shen, S. Wanwimolruk, M.S. Roberts, C.R. Clark, A sensitive assay for Aspirin and its metabolites using reversed-phase ion-pair high-performance liquid chromatography, J. Liquid Chromatogr. Rel. Technol. 13 (1990) 751-761.

[21] F. Kees, D. Jehnich, H. Grobecker, Simultaneous determination of acetylsalicylic acid and salicylic acid in human plasma by high-performance liquid chromatography, J. Chromatogr. B: Biomed. Sci. Appl. 677 (1996) 172-177.

[22] S.K. Bae, K.A. Seo, E.J. Jung, H.-S. Kim, C.-Q. Yeo, J.-H. Shon, K.-M. Park, K.-H. Liu, J.-G. Shin, Determination of acetylsalicylic acid and its major metabolite, salicylic acid, in human plasma using liquid chromatography-tandem mass spectrometry: application to pharmacokinetic study of Astrix ${ }^{\circledR}$ in Korean healthy volunteers, Biomed. Chromatogr. 22 (2008) 590-595. 
[23] R. Nirogi, V. Kandikare, K. Mudigonda, D. Ajjala, R. Suraneni, P. Thoddi, Simultaneous extraction of acetylsalicylic acid and salicylic acid from human plasma and simultaneous estimation by liquid chromatography and atmospheric pressure chemical ionization/tandem mass spectrometry detection, Arzneimittelforschung 61 (2011) 301-311.

[24] D.V. Bharathi, K.K. Hotha, P.R.R. Kolagatla, V. Venkateswarlu, Low dose aspirin estimation: an application to a human pharmacokinetic study, Biomed. Chromatogr. 27 (2013) 589-598.

[25] X. Xu, L. Koetzner, J. Boulet, H. Maselli, J. Beyenhof, G. Grover, Rapid and sensitive determination of acetylsalicylic acid and salicylic acid in plasma using liquid chromatography-tandem mass spectrometry: application to pharmacokinetic study, Biomed. Chromatogr. 23 (2009) 973-979.

[26] http://www.fda.gov/downloads/Drugs/Guidances/ucm070107.pdf

[27] http://www.ema.europa.eu/docs/en_GB/document_library/Scientific_guideline/2011/0 8/WC500109686.pdf

[28] J.R. Paterson, C. Blacklock, G. Campbell, D. Wiles, J.R. Lawrence, The identification of salicylates as normal constituents of serum: a link between diet and health?, J. Clin. Pathol. 51 (1998) 502-505.

[29] J.R. Paterson, G. Baxter, J.S. Dreyer, J.M. Halket, R. Flynn, J.R. Lawrence, Salicylic acid sans aspirin in animals and man: persistence in fasting and biosynthesis from benzoic acid, J. Agric. Food Chem. 56 (2008) 11648-11652.

[30] S.R. Polagani, N.R. Pilli, V. Gandu, High performance liquid chromatography mass spectrometric method for the simultaneous quantification of pravastatin and aspirin in human plasma: Pharmacokinetic application, J. Pharm. Anal. 2 (2012) 206-213.

[31] M. Rizk, M.S. Abdel-Rahman, Salicylamide reverses the aspirin-antagonistic effect of salicylic acid on rat platelet cyclooxygenase, Prostaglandins Leukotrienes and Essential Fatty Acids 51 (1994) 363-367. 
Table 1 Intra- and inter-day accuracy and precision data for ASP and SAL.

\begin{tabular}{|c|c|c|c|c|c|c|c|c|c|c|}
\hline & & Mean & & & Mean & & & Mean & & \\
\hline Analyte & conc. & $\begin{array}{c}\text { measured } \\
\text { conc. } \\
{[\mathrm{ng} / \mathrm{ml}]}\end{array}$ & $\begin{array}{c}\text { Accuracy } \\
{[\%]}\end{array}$ & $\begin{array}{c}\text { Precision } \\
\text { [\%] }\end{array}$ & $\begin{array}{c}\text { measured } \\
\text { conc. } \\
{[\mathrm{ng} / \mathrm{ml}]}\end{array}$ & $\begin{array}{c}\text { Accuracy } \\
{[\%]}\end{array}$ & $\begin{array}{c}\text { Precision } \\
\text { [\%] }\end{array}$ & $\begin{array}{c}\text { measured } \\
\text { conc. } \\
{[\mathrm{ng} / \mathrm{ml}]}\end{array}$ & $\begin{array}{c}\text { Accuracy } \\
{[\%]}\end{array}$ & $\begin{array}{c}\text { Precision } \\
\text { [\%] }\end{array}$ \\
\hline
\end{tabular}

\section{Intra-day}

$(n=6)$

Day 1

Day 2

Day 3

\begin{tabular}{|c|c|c|c|c|c|c|c|c|c|c|}
\hline \multirow[t]{4}{*}{ ASP } & 1.00 & 0.998 & -0.2 & 11.7 & 0.954 & -4.6 & 14.2 & 0.909 & -9.1 & 14.8 \\
\hline & 3.00 & 2.93 & -2.3 & 11.1 & 2.91 & -3.0 & 6.5 & 2.81 & -6.3 & 2.9 \\
\hline & 20.0 & 19.5 & -2.5 & 2.8 & 19.3 & -3.5 & 6.1 & 20.1 & 0.5 & 1.2 \\
\hline & 400 & 391 & -2.3 & 4.7 & 377 & -5.8 & 5.3 & 406 & 1.5 & 4.3 \\
\hline \multirow[t]{4}{*}{ SAL } & 80.0 & 78.4 & -2.0 & 6.1 & 75.2 & -6.0 & 10.0 & 84.1 & 5.1 & 5.3 \\
\hline & 240 & 243 & 1.3 & 5.6 & 233 & -2.9 & 5.1 & 236 & -1.7 & 2.5 \\
\hline & 800 & 776 & -3.0 & 2.8 & 797 & -0.4 & 4.7 & 753 & -5.9 & 1.0 \\
\hline & 6400 & 6100 & -4.7 & 1.5 & 6380 & -0.3 & 3.2 & 6180 & -3.4 & 3.7 \\
\hline
\end{tabular}

Inter-day

$(n=18)$

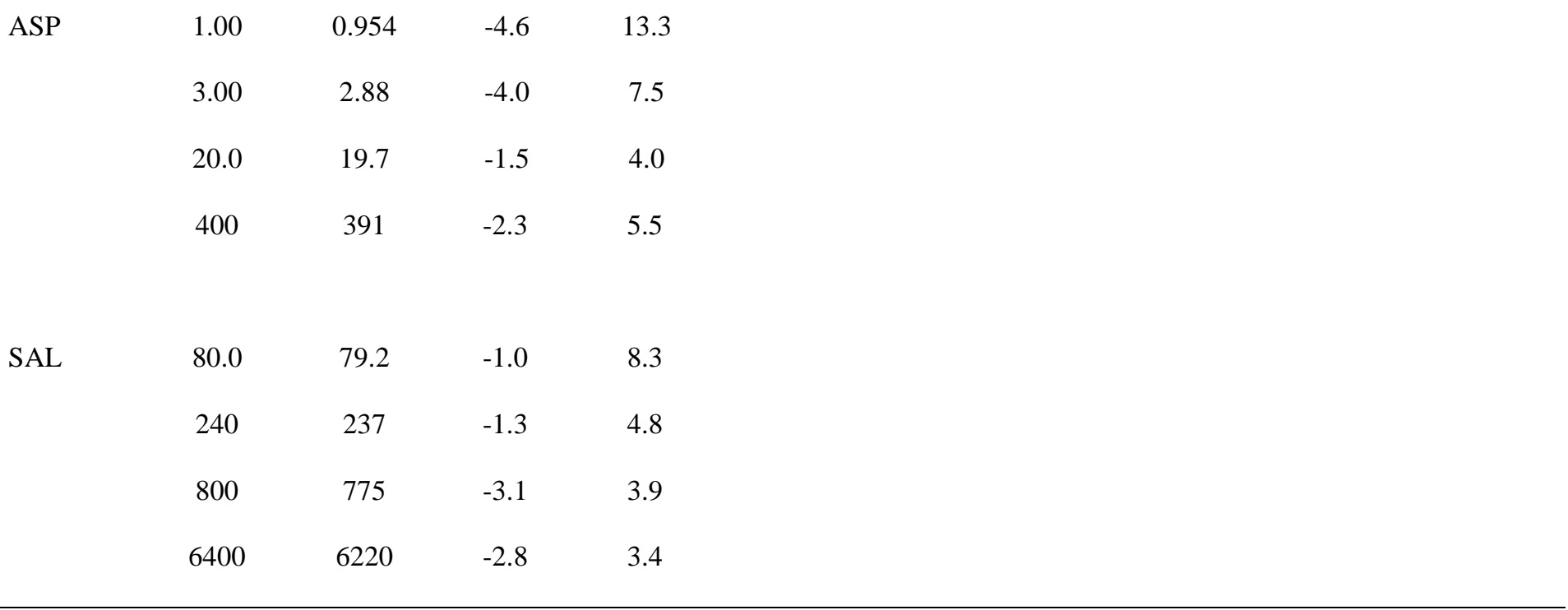


Table 2. The main results of stability tests for ASP and SAL

\begin{tabular}{|c|c|c|c|c|c|c|}
\hline Stability test & & ASP & & & SAL & \\
\hline & $\begin{array}{c}\text { Nominal } \\
\text { concentration } \\
{[\mathrm{ng} / \mathrm{ml}]}\end{array}$ & $\begin{array}{c}\text { Measured } \\
\text { concentration, } \\
\text { mean }[\mathrm{ng} / \mathrm{ml}]\end{array}$ & $\begin{array}{c}\text { Deviation } \\
\text { [\%] }\end{array}$ & $\begin{array}{c}\text { Nominal } \\
\text { concentration } \\
{[\mathrm{ng} / \mathrm{ml}]}\end{array}$ & $\begin{array}{c}\text { Measured } \\
\text { concentration, } \\
\text { mean }[\mathrm{ng} / \mathrm{ml}]\end{array}$ & $\begin{array}{c}\text { Deviation } \\
\text { [\%] }\end{array}$ \\
\hline 0 hour (initial) & & 2.95 & -1.7 & & 254 & 5.8 \\
\hline $\begin{array}{l}4 \text { hours in ice-water } \\
\text { bath }\end{array}$ & & 2.89 & -3.7 & & 252 & 5.0 \\
\hline Third freeze-thaw & 3.00 & 2.58 & -14.0 & 240 & 228 & -5.0 \\
\hline $\begin{array}{l}52 \text { hours in } \\
\text { autosampler at }+4{ }^{\circ} \mathrm{C}\end{array}$ & & 2.98 & -0.7 & & 253 & 5.4 \\
\hline 33 days below $-70^{\circ} \mathrm{C}$ & & 2.88 & -4.0 & & 238 & -0.8 \\
\hline 90 days below $-70^{\circ} \mathrm{C}$ & & 3.02 & 0.7 & & 243 & 1.3 \\
\hline 0 hour (initial) & & 379 & $\begin{array}{l}-5.3 \\
\end{array}$ & & 6030 & $\begin{array}{l}-5.8 \\
\end{array}$ \\
\hline $\begin{array}{l}4 \text { hours in ice-water } \\
\text { bath }\end{array}$ & & 371 & -7.3 & & 5827 & -9.0 \\
\hline Third freeze-thaw & 400 & 369 & -7.8 & 6400 & 5660 & -11.6 \\
\hline $\begin{array}{l}52 \text { hours in } \\
\text { autosampler at }+4{ }^{\circ} \mathrm{C}\end{array}$ & & 385 & -3.8 & & 6453 & 0.8 \\
\hline 33 days below $-70^{\circ} \mathrm{C}$ & & 388 & -3.0 & & 6000 & -6.3 \\
\hline 90 days below $-70^{\circ} \mathrm{C}$ & & 375 & -6.3 & & 5850 & -8.6 \\
\hline $\begin{array}{l}0 \text { hour in whole blood } \\
\text { (initial) }\end{array}$ & & 3.41 & & & 283 & \\
\hline $\begin{array}{l}1 \text { hour in whole blood } \\
\text { in ice-water bath }\end{array}$ & 3.00 & 3.15 & -7.6 & 240 & 282 & -0.4 \\
\hline $\begin{array}{l}0 \text { hour in whole blood } \\
\text { (initial) }\end{array}$ & & 408 & & & 7880 & \\
\hline $\begin{array}{l}1 \text { hour in whole blood } \\
\text { in ice-water bath }\end{array}$ & 400 & 454 & 11.3 & 6400 & 8042 & 2.1 \\
\hline
\end{tabular}


Table 3. The mean pharmacokinetic parameters for ASP and SAL

\begin{tabular}{cccccccccc}
\hline & \multicolumn{3}{c}{ ASP } & & & SAL & & \multicolumn{3}{c}{ SAL/ASP ratio } \\
& Mean & SD & CV\% & Mean & SD & CV\% & Mean & SD \\
& & & & & & & & \\
\hline Cmax $[\mathrm{ng} / \mathrm{ml}]$ & 696 & 421 & 60.5 & 4370 & 1230 & 28.1 & 6.28 & 2.92 \\
$\mathrm{AUC}_{(0-\mathrm{t})}[\mathrm{ng} * \mathrm{~h} / \mathrm{ml}]$ & 785 & 389 & 49.5 & 17800 & 5000 & 28.1 & 22.7 & 12.9 \\
\hline
\end{tabular}


Table 4. Pharmacokinetic parameters of volunteer-3 and volunteer-29

\begin{tabular}{ccc}
\hline Volunteer & 3 & 29 \\
\hline ASP & & \\
\hline Cmax $[\mathrm{ng} / \mathrm{ml}]$ & 1750 & 189 \\
$\mathrm{AUC}_{(0-\mathrm{t})}[\mathrm{ng} * \mathrm{~h} / \mathrm{ml}]$ & 1290 & 139 \\
\hline $\mathrm{SAL}$ & & \\
\hline $\mathrm{Cmax}[\mathrm{ng} / \mathrm{ml}]$ & 5350 & 4590 \\
$\mathrm{AUC}_{(0-\mathrm{t})}[\mathrm{ng} * \mathrm{~h} / \mathrm{ml}]$ & 16300 & 20500 \\
\hline
\end{tabular}


Figure captions:

Figure 1. Chemical structure of aspirin, salicylic acid and their deuterated analogues (aspirin-d4 and salicylic acid-d4)

Figure 2. Typical MRM chromatograms for ASP, SAL and ISTs of blank human plasma (A), LLOQ sample (B), ULOQ sample (C)

Figure 3. The average plasma concentrations of ASP and SAL (mean $\pm S D, n=33$ ) in volunteers administered with 100 mg Aspirin Protect

Figure 4. Individual pharmacokinetic curves of volunteer-3 and volunteer-29 


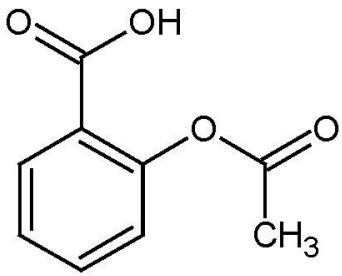

Aspirin

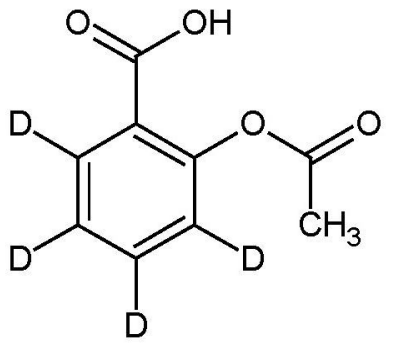

Aspirin-d4

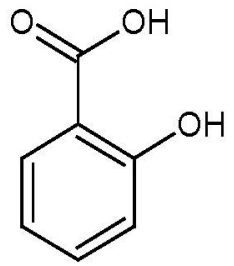

Salicylic acid

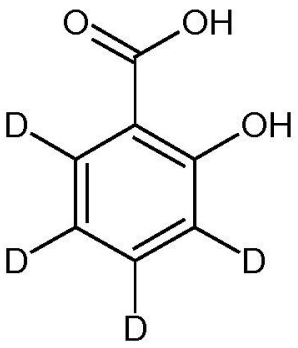

Salicylic acid-d4 

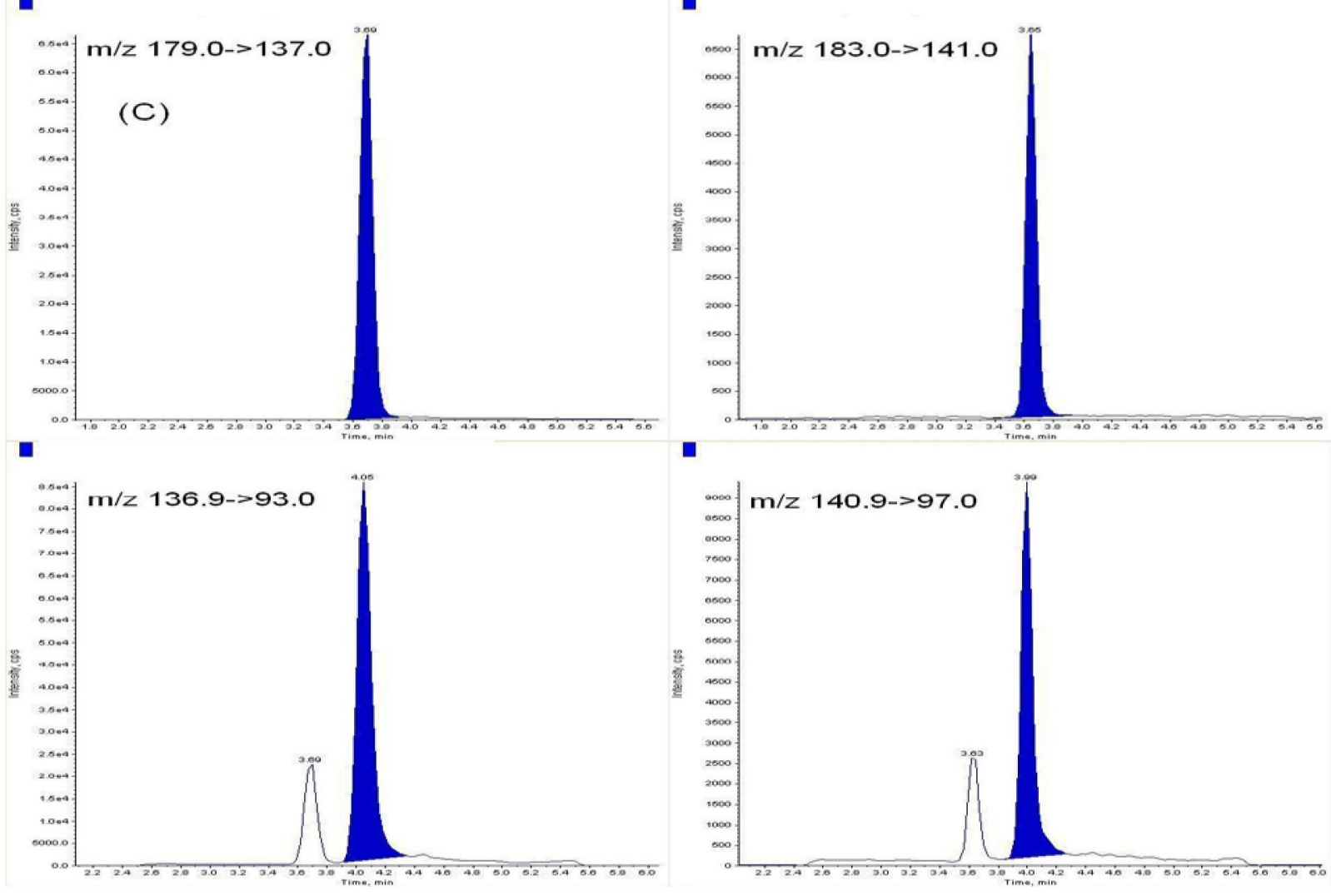


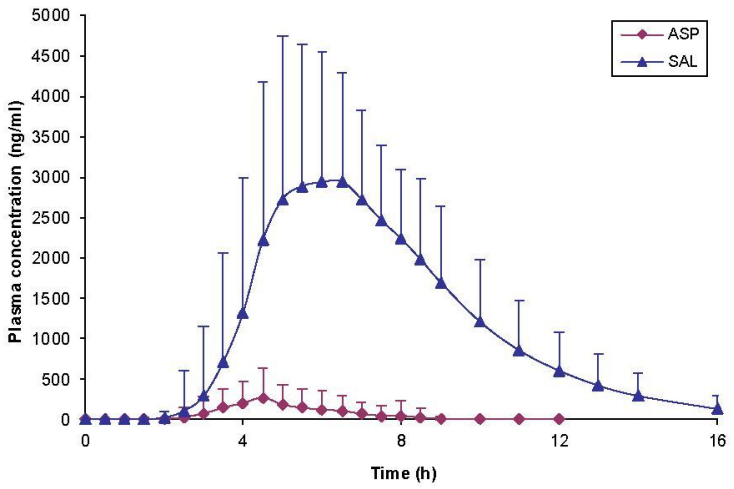



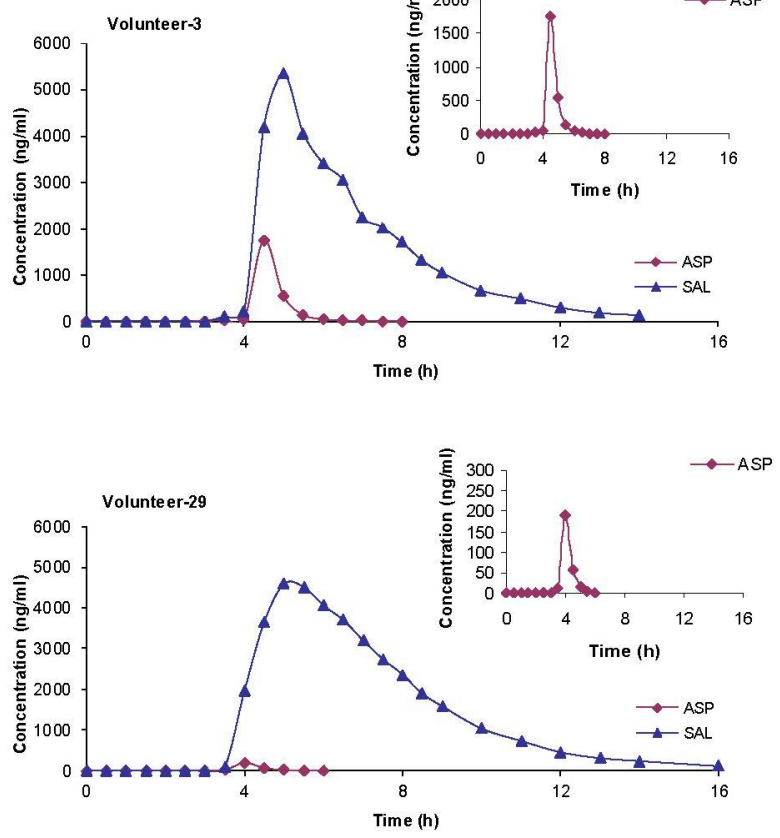